\section{Blockade of the distal sciatic nerve with the patient in the supine position using a newly developed position aid with integrated ultrasound probe holder}

\author{
Jens Magunia, Hans-Bernd Hopf and Michael Schütz* \\ Department of Anesthesia and Perioperative Medicine, Asklepios Klinik Langen, Langen, Germany
}

\section{Abstract}

Background: We have developed a femoral supporting pad with an integrated ultrasound probe holder and examined its practical usability on patients with lower limb surgery.

Objectives: To evaluate the function of this novel femoral supporting pad with respect to its practicability during the performance of a distal sciatic nerve blockade, the time needed to perform this blockade including the catheter insertion and the quality of postoperative analgesia within the first 24 hours.

Methods: 50 patients which had been scheduled for elective lower leg, ankle or foot surgery had received a continuous blockade of the distal sciatic nerve. Sciatic nerve blockade was performed sonographically controlled with the patients in supine position by using our novel femoral supporting pad with an integrated ultrasound probe holder. Primary endpoint: duration of the intervention. Secondary endpoints: pain intensity (visual analogue scale VAS 0-10) at the first postoperative day; cumulative opioid (piritramide) requirement during their stay on the post Anaesthesia care unit (PACU) with vs. without distal sciatic nerve blockade.

Results: $49 / 50$ patients received a distal sciatic catheter, which had been sonographically placed within a mean time (mean $\pm s d$ ) of 11:30 $\pm 3: 13$ minutes. VAS at the first postoperative day was (mean $\pm s d) 1 \pm 2$ at rest and $2 \pm 2$ as maximum. The piritramide requirement during PACU stay (mean $\pm \mathrm{sd}$ ) was $11 \pm 8 \mathrm{mg}$ without vs. $3 \pm 6 \mathrm{mg}$ with distal sciatic nerve blockade $(p<0.05)$.

Conclusion: Continuous distal sciatic nerve blockade using a novel femoral supporting pad with an integrated ultrasound probe holder was feasible in 49 of 50 patients within 11 minutes and 30 seconds.

\section{More Information}

Submitted: 03 July 2019

Approved: 08 July 2019

Published: 09 July 2019

How to cite this article: Magunia J, Bernd HB, Schütz M. Blockade of the distal sciatic nerve with the patient in the supine position using a newly developed position aid with integrated ultrasound probe holder. Int J Clin Anesth Res. 2019; 3: 003-006.

\section{DOI: 10.29328/journal.ijcar.1001012}

Copyright: (c) 2019 Magunia J, et al. This is an open access article distributed under the Creative Commons Attribution License, which permits unrestricted use, distribution, and reproduction in any medium, provided the original work is properly cited

Keywords: Distal sciatic nerve blockade; Ultrasound; Nerve blockade; Postoperative pain management; Patient positioning; Supine; Mount; Cradle; Femoral supporting pad; Sono; Sonographical; Pontinuos

ISSN: 2640-2866

Check for updates

\section{Introduction}

Ultrasound guided blockade of the distal sciatic nerve is a standard technique to provide intra-neural and postoperative analgesia for lower limb and knee surgery [1-4]. While ultrasound guidance might avoid intra-neural injection [5], the various techniques (anterior medial adductor canal approach, distal medial or lateral approach) are time consuming in their realization and might be awkward, when using ultrasound guidance, because of the need of special positioning of the patient or its leg [6-11]. Accordingly we have developed a novel femoral supporting pad with an integrated ultrasound probe holder to easily perform ultrasound guided distal sciatic nerve blockade while the patient remaining in the supine position. The present paper reports the results of the first clinical use of this device.

\section{Patients and Methods}

This study was a feasability study to evaluate our new developed femoral supporting pad with integrated ultrasound probe holder on patients undergoing lower limb surgery. After having obtained approval of the local ethical committee (Landesärztekammer Hessen FF 18/2018) and written informed consent, 50 patients which underwent elective lower 
leg-, ankle- or foot-surgery received a continuous blockade of the distal sciatic nerve. Exclusion criteria were: An age lower 18 years, rejection of the nerve blockade, ASA physical status $>3$, infection of the lateral thigh and allergy against the local anesthetic which were used for the blockade. The nerve blockade had been performed in general anesthesia (laryngeal mask or endotracheal tube after an induction dose of fentanyl, propofol and continuous administration of sevoflurane at $1 \mathrm{MAC}$ in $30-50 \% \%$ fraction of inspired oxygen mixed with ambient air).

The newly developed femoral supporting pad consisted of polylactide and polyurethane and was produced by a commercially available 3-D-printer (Prusa research, MK3, Czech Republic, Prague). It consists of two parts (Figure 1) which are inert for standard alcoholic based disinfection solutions. The ultrasound probe has to be inserted into the inlay (Figure 2) and must be covered with ultrasound gel (Medimex, Germany, Limburg). Thereafter the whole device has to be covered with a sterile transparent plastic drape (Ecolab, general purpose equipment drape, 25cm x 55xm, USA, Minnesota, St. Paul) on which a further portion of ultrasound gel has to be placed. Then the pad with the drape was placed under the midth of the thigh (Figure 3). By shifting the femoral supporting pad horizontally in the cranial, kaudal or lateral direction under the thigh, the optimum position to visualize the distal sciatic nerve could be localized. Sciatic nerve blockade was performed sonographically controlled in the supine position with the help of our novel femoral supporting pad with an integrated ultrasound probe holder by four consultant anesthesiologists. Ultrasound was performed with a linear ultrasound probe (6-15 MHz, HFL50, SII, FUJIFILM SonoSite) needle positioning had been performed with a 19 gauge stimulation needle set (NanoLine, 100mm, Pajunk, Germany). Stimulation was performed at $2 \mathrm{~Hz}$ and a motoric response at $0.5 \mathrm{~mA}$ with $0.1 \mathrm{~ms}$ impulse duration was accepted as sufficient. After identification of the distal sciatic nerve by dual guidance (ultrasound and stimulation) $15 \mathrm{ml}$ priloacaine $1 \%$ und $10 \mathrm{ml}$ ropivacaine $0.2 \%$ were injected (Figures 4 , 5). Thereafter under continuous ultrasound observation of the needle tip near the nerve, a catheter (SonoLong, Pajunk, Germany) was placed with its tip $2 \mathrm{~cm}$ beneath the nerve. Finally, to verify the catheter position, $1 \mathrm{ml}$ of air was injected through the catheter (Figure 6). Continuous postoperative analgesia was provided by infusion of ropivacaine $0.2 \%$ via the catheter with a flow rate of $4-8 \mathrm{ml} / \mathrm{h}$ dependent on the patient's VAS score. The primary endpoint was the duration of the whole intervention including skin disinfection. We defined the preparation of the probe holder with ultrasound gel as the beginning of the whole procedure and the fixation of the sciatic catheter to the skin as the end of the procedure. Secondary endpoints were the pain intensity (VAS, 0-10) at the first postoperative day and the cumulative piritramide requirement during the stay in the PACU.

\section{Statistics}

The number of patients that were needed to find a

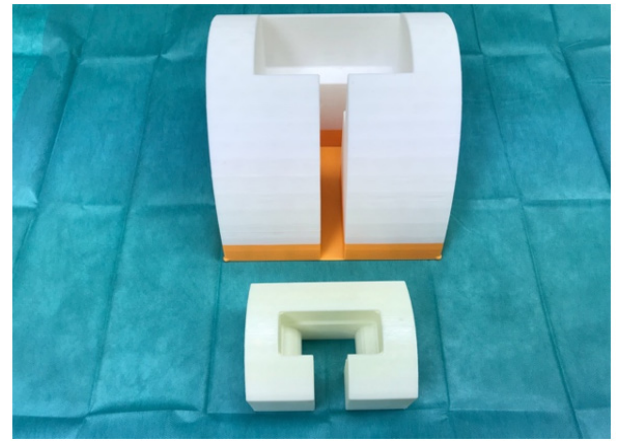

Figure 1: Position aid and the integrated ultrasound probe holder.

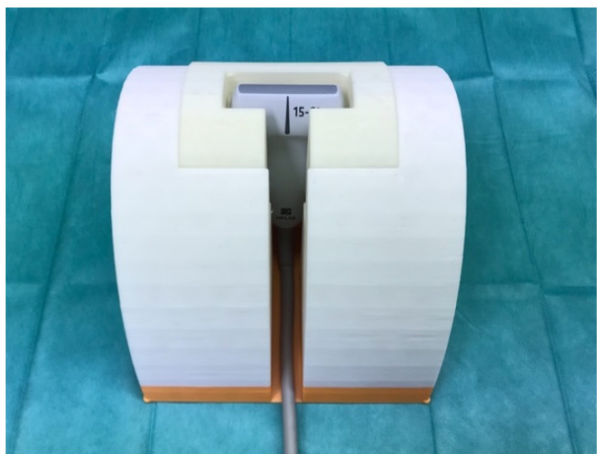

Figure 2: Ultrasound probe in the probe holder inserted into the position aid

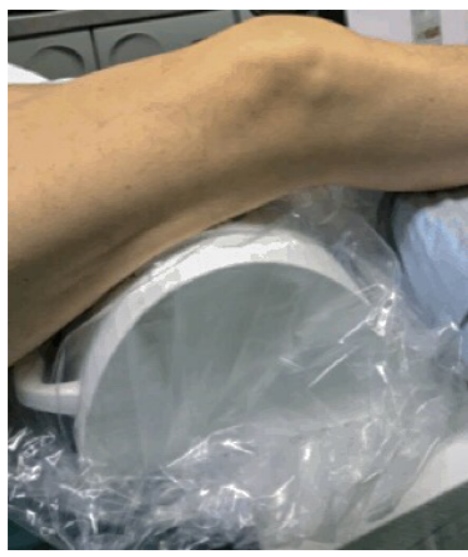

Figure 3: Position aid with inserted probe (see Figure 2) placed under the thigh.

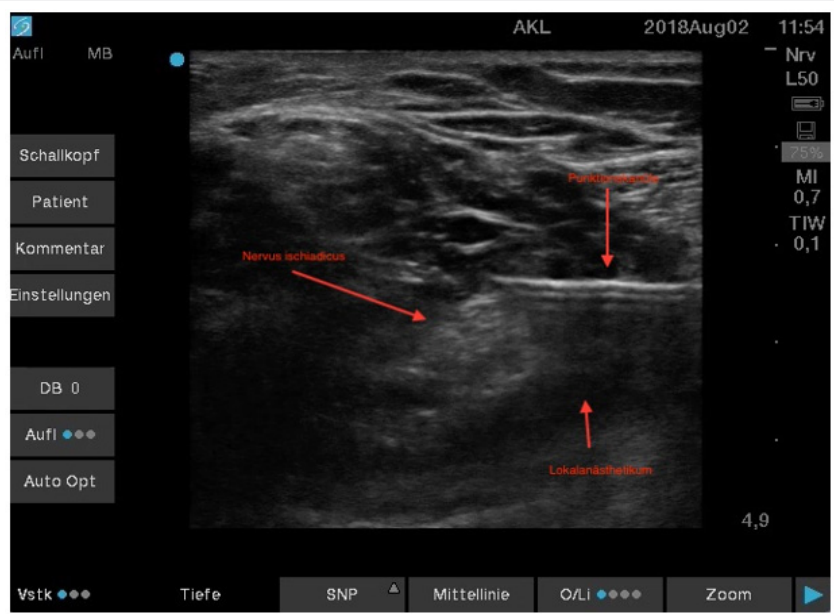

Figure 4: Distal sciatic nerve with puncture needle in place just after injection local anesthetic solution. 


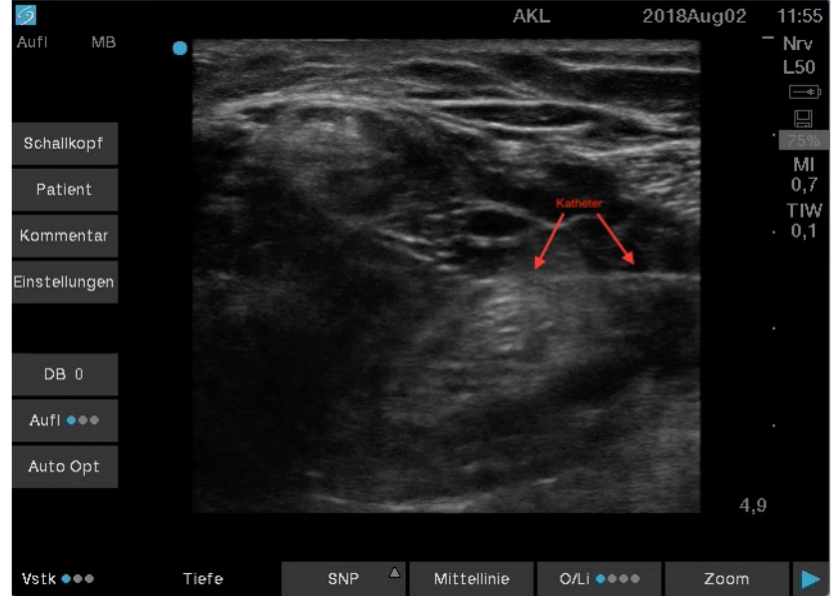

Figure 5: Sciatic nerve with the catheter in place.

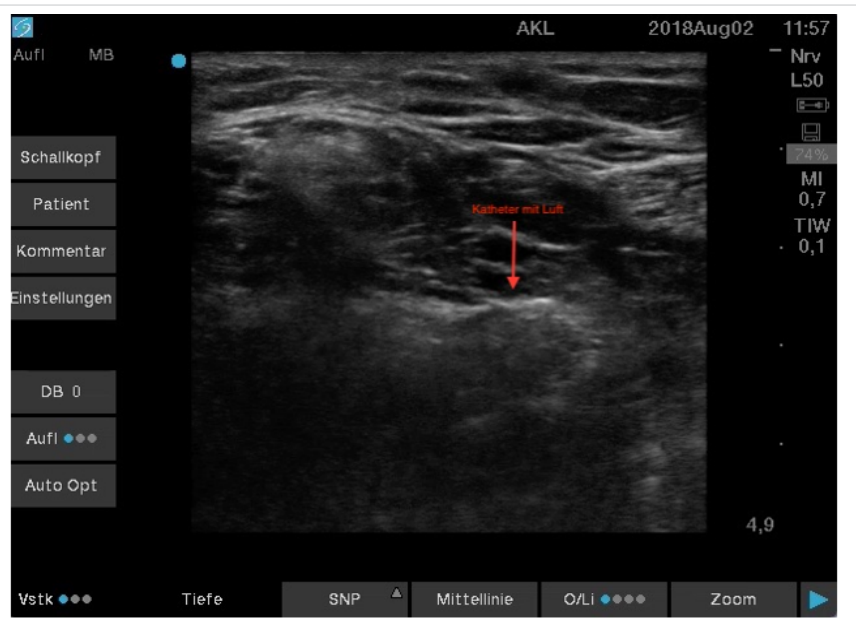

Figure 6: Control of catheter placement with $1 \mathrm{ml}$ of air injected into the catheter.

difference in time needed by each anesthesiologist to perform the blockade, had been calculated with aid of the institute of biostatistics and mathematically modelling of the Goethe University Frankfurt/Germany. Assuming that four anesthesiologists would perform the blockade by using the femoral supporting pad, 50 patients would be needed to show a difference in time between the four anesthesiologists. Pain intensity was assessed by pain nurses at the first postoperative day by using the VAS. To get additional data about the quality of the sciatic nerve blockade, the cumulative intravenous piritramide requirement during the PACU stay had been compared to the cumulative intravenous piritramide requirement during the PACU stay of 75 comparable patients (50 females, 25 males) having undergone identical lower limb surgical procedures during the year 2017 without distal sciatic nerve blockade. Demographic data of those 75 patients showed no significant difference compared to the 50 patients of 2018. All data are presented as mean \pm standard deviation and confidence intervals if not otherwise stated. Comparison of cumulative piritramide requirement during the PACU stay between the patients with sciatic nerve blockade versus those without sciatic nerve blockade had been performed with the Whitney-Mann-U-Test. A p-value below 0.05 had been considered significant.

\section{Results}

We studied 50 patients, 24 female, 26 male. The mean age was $61+/-18$ years, mean height was $172+/-9 \mathrm{~cm}$ and mean body weight was $80+/-14 \mathrm{~kg}$. Mean BMI was 27 . Sonographic visualization and localization of the sciatic nerve was possible in $49 / 50$ patients. In one patient sonographic identification of the sciatic nerve failed. In that patient, localization was solely accomplished by nerve stimulation. Three of the four anesthesiologists treated 12 patients, one treated 14 patients. There was no difference in time needed to perform the blockade between the four anesthesiologists (No. 1 10:31, No 2 9:54, No 3 10:34, No. 4 12:13; all numbers given in minutes and seconds). Overall mean time for the whole procedure of the four anesthesiologists was 11 minutes 30 seconds +/- 3 minutes 13 seconds. Lowest mean pain intensity at the first postoperative day on the VAS scale was 1 at rest, maximum pain intensity was 2.18 patients experienced no pain, while in 36 patients maximum pain intensity was 3 (Figure 7). One patient experienced a pain intensity of 6 at rest and 9 as pain maximum. In that patient sonographical identification of the distal sciatic nerve had failed. Compared to the cumulative PACU piritramide requirement of the 75 patients having undergone identical lower limb surgical procedures in the year 2017, the cumulative piritramide requirement during the PACU stay had been reduced from 11 +/- $8 \mathrm{mg}$ in 2017 to 3 +/$6 \mathrm{mg}$ in 2018. In 201820 out of 50 patients with distal sciatic nerve blockade required intravenous piritramide during the PACU stay, in contrast to 69 / 75 patients without distal sciatic nerve blockade in 2017 ( $\mathrm{p}<0.05$ / 2018 vs. 2017).

\section{Discussion}

The main result of this pilot study is, that the distal sciatic nerve can be easily identified and blocked by using the novel femoral supporting pad with integrated ultrasound probe holder. In fact, visualization, identification and placement of a catheter to the distal sciatic nerve was accomplished in 49/50 patients in around 12 minutes. For several reasons our femoral supporting pad with the integrated ultrasound probe holder is a substantial improvement compared to the techniques used currently. First, compared to the various described techniques [6-10], which are used for the blockade of the distal sciatic nerve, the patient can remain in supine

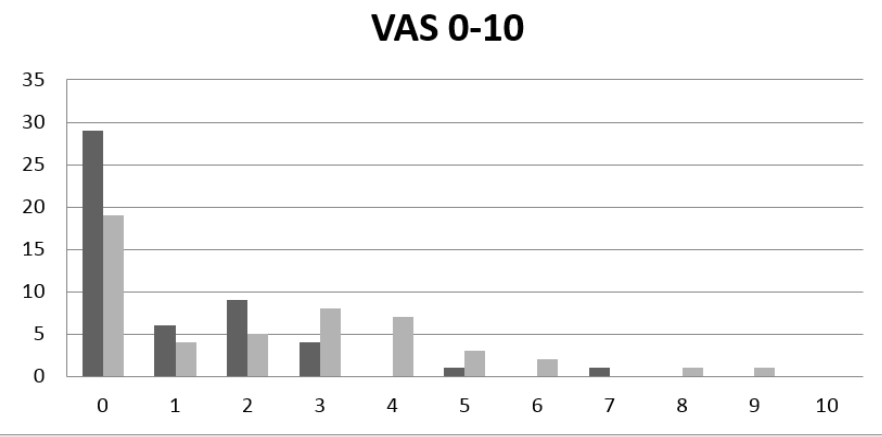

Figure 7: VAS scores at the first postoperative day. X-axis: VAS-score, $y$-axis: number of patients; black pillars: pain at rest; grey pillars: maximum pain. 
position, while the femoral supporting pad is placed under the thigh and shifted along the length axis until an optimal visualization of the distal sciatic nerve has been achieved. Second, with our integrated ultrasound probe holder a continuous visualization of the needle and its tip on its way to the nerve is possible. Thus, both hands of the anesthesiologist performing the blockade are free to direct the needle during the procedure as well as the catheter placement near to the sciatic nerve. Third, with the weight of the thigh lying on our femoral supporting pad the distance between the probe and the thigh is diminished thus enabling a much better ultrasound imaging of the structures of the thigh in contrast to the hand held ultrasound probe, which has to be pushed manually against the thigh. Finally, the integrated ultrasound probe holder enables a far better teaching of residents, since it attains a continuous visualization of the needle tip and catheter without the need of frequent manual reposition maneuvers with resultant decreasing performance of the ultrasound technique [12]. In fact our mean intervention time below 12 minutes compares favourable to published data comparing ultrasound vs. stimulator-guided sciatic nerve blockade [13]. However, we cannot exclude that the technique might be somewhat more difficult and therefore a little bit more time consuming in patients with greater diameters of the thigh compared to the patients treated in the present study. While the integrated ultrasound probe holder is a considerable improvement for needle and catheter direction compared to the manually hold probe, one disadvantage is the fixed angle of the probe. While shifting the thigh in the cranio-kaudal and lateral directions is possible even after having started with the puncture, it is not possible to change the angle of the probe in order to get a better ultrasound imagine. Thus, it may be difficult or impossible in some patients to identify the sciatic nerve. In fact, this was the case in one patient in our pilot series. Accordingly we suggest to always use dual guidance (both ultrasound imagine and electrical stimulation) to identify the nerve. The quality of postoperative analgesia as evaluated on the first postoperative day was excellent, with $18 / 50$ patients having a VAS of zero both at rest and as maximum pain and $36 / 50$ patients having a VAS of equal or less than 3. This is in accordance with recent data in the literature showing the superiority of ultrasound guided nerve blocks compared to a stimulation technique solely [2]. In addition, the cumulative piritramide consumption during the PACU stay in the 50 patients with a distal sciatic nerve block compared to 75 matched patients from 2017 without, was significantly reduced by $70 \%$, also showing the excellent quality of the blockade. In summary continuous distal sciatic nerve blockade using a novel femoral supporting pad with an integrated ultrasound probe holder was possible in 49 of
50 patients in less than 12 minutes and provided excellent postoperative analgesia.

\section{References}

1. Blunk JA. Ultraschall in der interventionellen Schmerztherapie. Anaesthesist. 2013; 62: 931-946.

2. Caio X, Zhao X, Xu J, Liu Z, Li Q. Ultrasound-guided technology versus neurostimulation for sciatic nerve block: a Meta-analysis. Int J Clin Exp Med. 2015; 8: 273-280.

PubMed: https://www.ncbi.nlm.nih.gov/pubmed/25784997

3. Cappelleri G, Ambrosoli AL, Gemma M, Cedrati VLE, Bizzarri F, et al Intraneural ultrasound-guided sciatic nerve block. Anesthesiology. 2018; 129: 241-248.

PubMed: https://www.ncbi.nlm.nih.gov/pubmed/29762181

4. Kienecker K. Kontinuierliche periphere Nervenblockade: Effektivere Maßnahmen zur postoperativen Schmerztherapie als die systemische Analgesie? Anaesthesist 2007; 56: 398-400.

5. Sala Blanch X, López AM, Carazo J, Hadzic A, Carrera A, et al Intraneural injection during nerve stimulator-guided sciatic nerve block at the popliteal fossa. $\mathrm{Br} \mathrm{J}$ Anaesth. 2009; 102: 855-61. PubMed: https://www.ncbi.nlm.nih.gov/pubmed/19420006

6. Danelli G, Fanelli A, Ghisi D, Moschini E, Rossi M, et al. Ultrasound vs nerve stimulation multiple injection technique for posterior poplitea sciatic nerve block. Anaesthesia. 2009; 64: 638-642. PubMed: https://www.ncbi.nlm.nih.gov/pubmed/19453318

7. Guntz E, Herman P, Debizet E, Delhaye D, Coulic V, et al. Sciatic nerve block in the popliteal fossa: description of a new medial approach. $J$ Can Anesth. 2004; 51: 817-882.

PubMed: https://www.ncbi.nlm.nih.gov/pubmed/15470171

8. Hadzic A, Vloka JD. A comparison of the posterior versus lateral approaches to the block of the sciatic nerve in the popliteal fossa. Anesthesiology. 1998; 88: 1480-1486.

PubMed: https://www.ncbi.nlm.nih.gov/pubmed/9637640

9. Mariano ER, Loland VJ, Sandhu NS, Bishop M, Lee D, et al. Comparative efficacy of ultrasound-guided and stimulating popliteal-sciatic perineural catheters for postoperative analgesia. J Can Anesth. 2010; 57: $919-926$

PubMed: https://www.ncbi.nlm.nih.gov/pubmed/20700680

10. Neuburger $M$, Schwemmer U, Volk T, Gogarten W, Kessler $P$, et al Lokalisation peripherer Nerven. Anaesthesist. 2014; 63: 422-428.

11. Osaka Y, Kashiwagi M, Nagatsuka Y, Miwa S. Ultrasound-guided media mid-thigh approach to sciatic nerve block with a patient in a supine position. J Anesth. 2011; 25: 621-624.

PubMed: https://www.ncbi.nlm.nih.gov/pmc/articles/PMC3152704/

12. Cataldo R, Carassiti M, Costa F, Martuscelli $M$, Benedetto $M$, et al Starting with ultrasonography decreases popliteal block performance time in inexperienced hands. BMC Anesthesiol 2012; 12: 33. PubMed: https://www.ncbi.nlm.nih.gov/pubmed/23253617

13. Van Geffen GJ, Van den Broek E, Braak GJJ, Giele JP, Gielen MJ, et al A prospective randomised controlled trial of ultrasound guided versus nerve stimulation guided distal sciatic nerve block at the popliteal fossa. Anaesth Intensive Care 2009; 37: 32-37.

PubMed: https://www.ncbi.nlm.nih.gov/pubmed/19157343 